\title{
Structural and enzymatic aspects of LMWPTP from Vibrio cholera 0395
}

\author{
Shramana Chatterjee $^{1}{ }$ Seema Nath $^{1}$, Udayaditya Sen $^{1}$ \\ ${ }^{1}$ C\&MB Division, Saha Institute Of Nuclear Physics, Kolkata, India \\ E-mail: shramana.chatterjee@saha.ac.in
}

Protein phosphorylation on tyrosine residues has been considered as a post translational modification on eukaryotes and also in bacteria (gram negative, gram positive) which creates recognition motifs for protein intercalation and cellular localization that affects the protein stability and regulate enzymatic activity. Protein tyrosine phosphatases (PTP) are a group of enzyme that removes the phosphate groups from phosphorylated tyrosine residues. Depending on the mechanism it can be classified into four subgroups, where class II contains only Low Molecular Weight Protein Tyrosine Phosphatase (LMWPTP).

The overall structure of this type of cytoplasmic ubiquitous Low molecular weight PTPase $($ Mr $\sim 18,000)$ contains Rosmann fold along with signature motif that forms a phosphate binding site equivalent to the P-loop. Both eukaryotic and prokaryotic LMWPTPs form dimer with a high dissociation constant in vitro involving their active site tyrosine residues of YYloop and become catalytically inactive [1]. It has been suggested that the inactive oligomers acts as 'supramolecular proenzyme' although its physiological congruity remains undefined. Among the PTPases, LMWPTPs have a unique feature of hosting two cysteines in the active site P-loop. The presence of second cysteine is believed to participate in disulphide bridge formation with the catalytic cysteine as partner, when the oxidative stress inside the cell increases. The disulphide bond is, however, reversibly broken down under reducing condition. Thus the second cysteine serves to protect the catalytic cysteine, which is prone to oxidation because of its microenvironments, from producing irreversibly oxidized suphenic/sulphonic acid derivatives.

Here we describe the $2.5 \AA$ crystal structure of Vibrio cholerae LMWPTP 2 (Vc-LMWPTP2) with substrate analogue MOPS bound at the active site. Tertiary structure analysis clearly indicates that Vc-LMWPTP2 forms a dimer. The dimeric state, also present in solution, is catalytically active indicating that the active site is accessible to substrate. Crystal structure demostrate also that dimerisation do not occlude the active site. The mode of dimerization of Vc-LMWPTP2 is, however, different from its homologue Vc-LMWPTP1 reported earlier [2]. Kinetic study using p-NPP shows that WT Vc-LMWPTP2 exhibit high catalytic activity but the double mutant where both the cysteines in the active site have been mutated to serine, exhibit nominal catalytic activity, suggesting the active participation of both the cystines in the catalytic mechanism. Role of several other mutants were decipher through kinetic studies. The surface charge distribution around the catalytic 'P-loop', that is believed to govern the specificity of LMWPTPS, is quite different in Vc-LMWPTP2 compared to the other reported LMWPTPS, physiological significance of which is driven by its selection of substrate binding.

[1] Zhang, M. et al (1997) Biochemistry, 36, 15-23.

[2] Nath, S. et al (2014) Biochemical and Biophysical Research Communications, 450, 390-395.
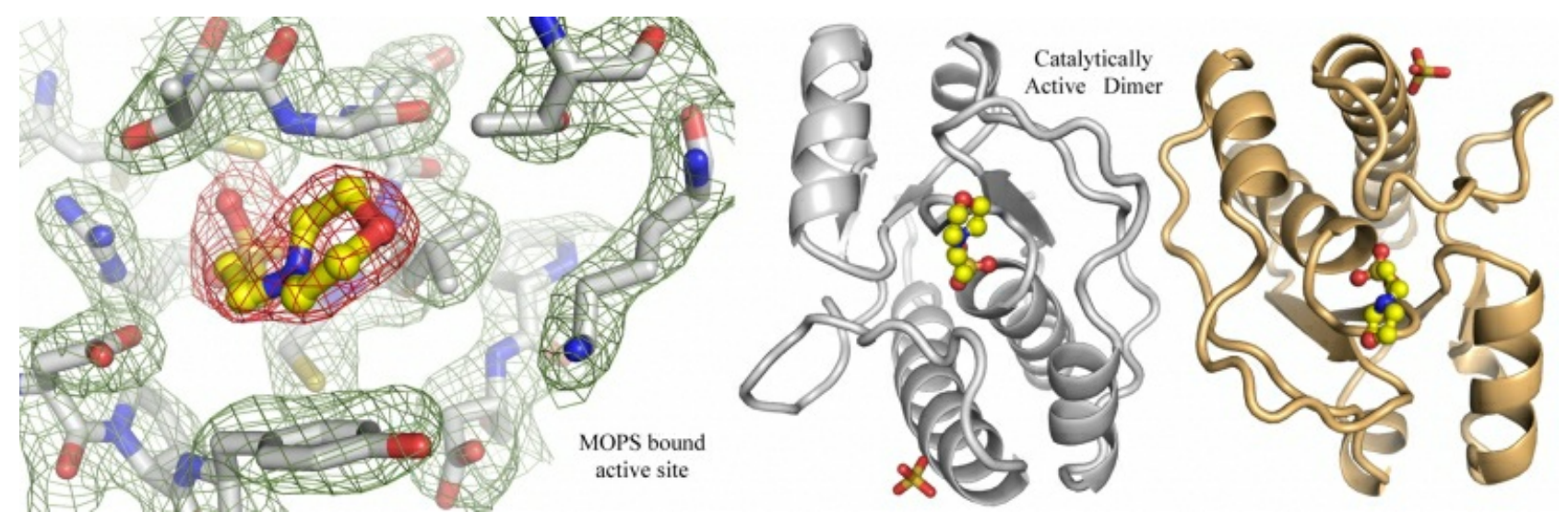

Keywords: Low Molecular weight protein tyrosine phosphatase, X-ray crystallography, Active dimer 